\title{
Management of 'In-Field' Skin Toxicity in Head and Neck Cancer Patients Treated with Combined Cetuximab and Radiotherapy
}

\author{
Domenico Cante $^{a} \quad$ Maria Rosa La Porta ${ }^{a}$ Pierfrancesco Franco ${ }^{b}$ Piera Sciacero $^{a}$ \\ Giuseppe Franco Girellia Annamaria Marra ${ }^{a}$ Gianmauro Numico ${ }^{c}$ \\ Nerina Denaro $^{d}$ Elvio Grazioso Russi ${ }^{\mathrm{e}} \quad$ Umberto Ricardi $^{f}$

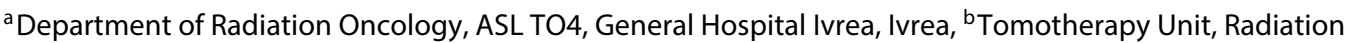 \\ Oncology Department, and 'Medical Oncology Department, Regional Hospital U. Parini, AUSL Valle d'Aosta, Aosta, \\ ${ }^{\mathrm{d}}$ Department of Oncology, AOU G. Martino Messina University, Messina, ${ }^{\mathrm{e}}$ Department of Radiation Oncology, AO \\ S. Croce e Carle, Cuneo, and ${ }^{f}$ Radiation Oncology Unit, Department of Medical and Surgical Sciences, University of \\ Turin, San Giovanni Battista Hospital, Turin, Italy
}

\section{Key Words}

Cetuximab - Epidermal growth factor receptor $\cdot$ Head and neck cancer $\cdot$ Radiation-induced toxicity $\cdot$ Dermatitis

\begin{abstract}
It is well known that the cetuximab (Cet) epidermal growth factor receptor (EGFR) antibody enhances the sensitivity of tumour cells to radiation, and it is likely that the concurrent administration of Cet and radiotherapy (RT) results in some degree of interplay between the effects of the individual agents on the skin and in the exacerbation of reactions normally seen with these individual agents. In this paper, we present a concise review of Cet/RT-related skin toxicity, focusing on mechanisms and pathogenesis, clinical presentation and scoring systems and, finally, therapeutic management.

(c) 2013 S. Karger AG, Basel
\end{abstract}

\section{Introduction}

It is common knowledge that the antibody against the epidermal growth factor receptor (EGFR), namely cetuximab (Cet; Merck-Serono, Darmstadt, Germany),

\section{KARGER}

E-Mail karger@karger.com www.karger.com/ocl enhances the sensitivity of tumour cells to radiation, and it has also been established that the concurrent administration of Cet and radiotherapy (Cet/RT) might result in some degree of interplay between the effects of the individual agents on the skin, resulting in the possible exacerbation of the usual reaction experienced with the individual agents given as exclusive therapeutic choice $[1,2]$. The pivotal phase III trial by Bonner et al. $[3,4]$ demonstrated that the addition of Cet to RT results in a significant increase in locoregional control and survival compared to RT alone, even on a long-term basis.

Moreover, the same trial did not show any statistically significant difference in terms of radiation dermatitis (either all grades or grade $\geq 3$ ) between the two treatment arms, although a 5\% trend towards an increase in the incidence of grade $\geq 3$ radiation dermatitis was detected in the Cet arm [3]. Conversely, some authors report severe radiation-induced dermatitis-like skin reactions with the combination of Cet/RT [5-7]. We herein present a concise review of 'in-field' Cet/RT-related skin toxicity with a focus on pathogenesis, clinical presentation and scoring systems and, finally, therapeutic management. 


\section{Materials and Methods}

During April 2013, a non-systematic literature review of Cetinduced dermal toxicity associated with RT in head and neck cancer patients was performed. The electronic databases used were: PubMed, The Cochrane Library, Scopus and Embase. The following keywords were combined: dermatitis, cetuximab; EGFR; radiotherapy; radiation; head and neck cancer. The electronic search results were supplemented by manual examination of reference lists from selected articles. The investigation focused on mechanisms and pathogenesis, clinical presentation, scoring systems, and therapeutic management. The literature search was limited to articles in English and to human patients. We analysed and discussed the literature, taking into account the previously reported reviews on this subject.

\section{Cet/RT Interaction on Skin Toxicity: Pathogenesis}

In-field skin reactions observed after Cet/RT therapeutic association present pathophysiological and clinical differences compared to those observed after exclusive radiation, since they are characterized by early onset, rapid resolution and usually no evidence of scars $[5,8]$. Considering Cet/RT association, the combination of skin responses to individual agents leads to a peculiar clinical presentation.

RT primarily injures the proliferating basal layer of the epidermis, causing cell depletion and consequent alteration in the physiological cell turnover. Granular cells and anucleated corneocytes show less radiosensitivity whereas dermal inflammatory response cells and the peripheral nervous system are activated by the release of alarmins and cytokines $[9,10]$, provoking dermatitis (i.e. oedema) and local hypersensitiveness (pruritus, erythema, pain). The final result is an inflamed derma covered by a thinned epidermis.

Epidermal thinning is mostly due to the stratum granulosum, involved in the production of lipids and natural moisturizing factors [11]. This stratum cooperates with the corneal stratum in the prevention of transepidermal water loss $[2,11]$. The damage is visible as clumps of exfoliated corneocytes, characteristic of dry desquamation associated with grade 1 radiation dermatitis reaction.

For high radiation doses, an almost complete loss of the basal cell, associated with a disruption of the basement membrane, might occur, leading to exposure of the inflamed [9] dermis and eventually to moist desquamation, described as grade $2-3$ reactions. Finally, skin necrosis or ulceration of the full-thickness dermis (grade 4 reactions) might occur, even though anecdotally, during the acute phase.
The EGFR signalling pathway plays an integral part in the development and maintenance of the normal skin, regulating survival, migration and proliferation of the various types of epidermis cells [12-14]. In vitro data show that EGFR inhibitors (EGFRis) reduce the proliferative activity and migration ability within the basal cells, concurrently inducing early cellular differentiation and apoptosis $[5,12]$. Thus, EGFR inhibition leads to a thinning of the functional strata $[12,15]$, favouring the development of xerosis $[13,16]$. Furthermore, the blockade of the EGFR induces a deranged chemokine expression in keratinocytes, leading to enhanced skin inflammation which is responsible for folliculitis and dermatitis $[17,18]$. Finally, it has been shown that EGFRis can alter the injury-induced innate immune response [19], compromising the synthesis of antimicrobial peptides [16] and favouring microbial colonization and superinfection [20].

\section{Clinical Manifestations}

Concurrent administration of Cet/RT might result in an exacerbation of skin reactions typical of individual agents, and interactions might manifest as a thinning of the epidermis, leading to a more frequent occurrence of microinjuries, marked xerosis and more intense inflammatory response. Necrosis has been described as focal and superficial $[13,15]$.

Thus, the reinforced Cet/EGFRi inflammatory effect favours the production of an inflammatory exudation which, in consequence of the altered transepidermal water loss barrier, dries off rapidly to form crusting exudates. These crusts might adhere to the skin, cause damage and provoke bleeding and/or pain [21]. The reparative capacity of damaged skin might also be compromised, rendering the cutis itself prone to abrasion. Finally, crusts may harbour bacteria, thereby potentially increasing the risk of superinfection, possibly exacerbated by the EGFRi deregulation effect on inflammatory immune reactions.

The type of reaction observed following concurrent Cet/RT depends on the degree of interaction between the two treatments; the clinical manifestations vary from a combination of dry/moist desquamation, mainly related to RT, and xerosis, inflammation and innate immune defence deregulation, associated with EGFRis. Whenever Cet is associated with RT, crusting exudation (sometimes associated with 'spontaneous' or 'minor trauma' bleeding; fig. 1) becomes evident mostly within in-field cutaneous areas. 


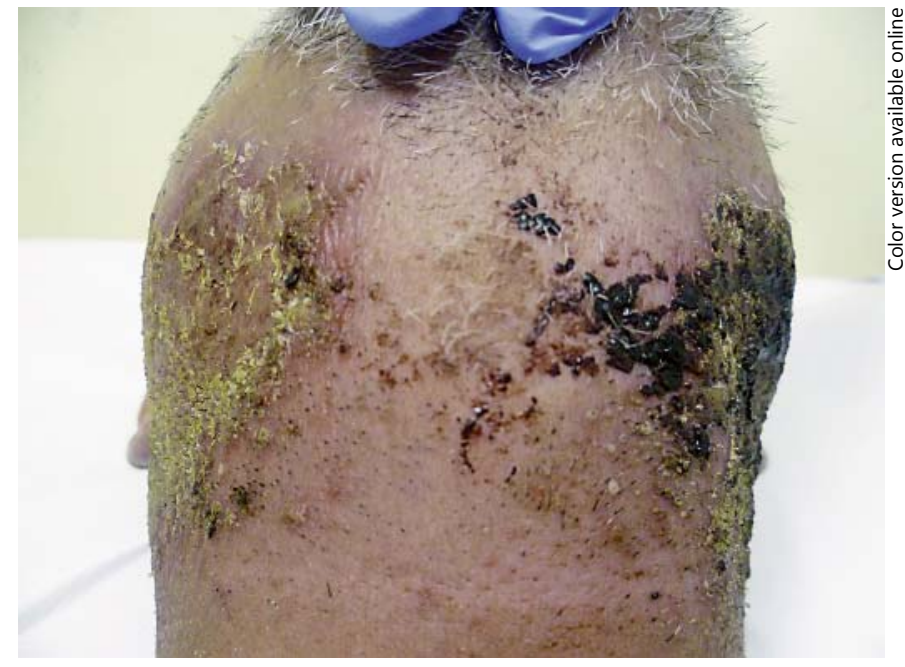

Fig. 1. In-field toxicity in a patient submitted to Cet and RT (60 Gy). While a yellow crusted exudate is visible on the right side, bloody scabs on the patient's left side are probably the consequence of traumatisms due to the fact that the patient used to sleep with his right cheek on the pillow.

During Cet/RT, the onset of radiation dermatitis may be earlier than with RT alone [15, 22], and the overall entity of toxicity may be more severe in terms of dermatitis [23]; however, patients generally recover within 1-2 weeks after treatment, even in the presence of crusts $[13,24,25]$.

Specifically, Cet might induce systemic skin toxicity: skin rash, skin dryness, xerosis, pruritus, hair abnormality, and nail changes $[25,26]$. The most common skin toxicity is a papulopustolar rash, which affects $60-80 \%$ of patients. It is generally mild to moderate, though severe (G3-4) in $5-20 \%$ of patients $[25,27]$. Incidence and entity are usually dose related [27], and generally, it is reversible, with complete resolution within 4 weeks after treatment discontinuation or, in some cases, even during maintained treatment; it may relapse or worsen at treatment restart. Papulopustular eruption consists of erythematous follicular papules which may potentially evolve into pustules. Lesions may coalesce into plaques covered by yellow crusts [21]. Frequent infection of these lesions has been described [20]. Skin xerosis is present in up to $35 \%$ of patients receiving EGFRis; it develops over time, particularly in areas previously or simultaneously affected with papulopustolar eruption [24].

Xerosis is often more widespread than skin rash; it may evolve into chronic asteatotic eczema, with erythema and worsening of pruritus. Xerosis and eczematous changes in the fingertips, palms and soles are usually associated with painful fissures.

\section{Scoring Systems of Dermatological Adverse Events}

The National Cancer Institute Common Terminology Criteria for Adverse Events version 4.3 (NCI-CTCAE version 4.3) is an established tool for grading radiation dermatitis [28].

Whereas it has proved suitable for grading dermatitis in patients receiving either RT or Cet, it is less appropriate for grading the type of dermatitis seen in patients receiving both Cet/RT. Indeed, the grading of radiodermatitis is often not applicable to in-field dermatitis as it does not include the associated adverse events of biological treatments. On the other hand, the grading of the systemic biological treatment effect, which includes the percentage of extension over the whole body skin, is not applicable when the reactions are confined to a limited skin surface such as in the irradiated field.

Thus, even though version 4.3 has either an appropriate systemic EGFRi (skin rash) or a radiation dermatitis toxicity classification [29], presently, there is no other grading system assessing the in-field severity of adverse events due to the association of biotherapy and RT.

These issues can explain the different bioradiotherapy in-field toxicity rates reported in different studies [4]. Indeed, severity has often been over- or underestimated [4], and a common treatment strategy could be difficult to set up.

This may compromise the appropriate management of skin reactions and, more importantly, may lead to treatment delay or interruption, thereby potentially adversely affecting outcome results.

There is general agreement that a clear and objective nomenclature for grading Cet-related radiation dermatitis is still lacking $[21,26,30,31]$. To our knowledge, the only scale that tries to overcome this limitation has been the one proposed by Bernier et al. [13]. These authors suggested modifications to the current NCI-CTCAE version 4.3 grading system and proposed grade-specific management strategies for patients developing radiation dermatitis during treatment with Cet/RT [13]. Together with radiation dermatitis events, the extension and severity of crusting lesions contribute to grade adverse events.

Probably, this first attempt has few limitations related to the fact that it does not take into account other specific biological treatment-related effects such as folliculi- 
tis, oedema, local and systemic inflammation. More recently, a more comprehensive classification has been proposed by a panel of experts [32].

\section{General Prophylactic Measures and Management of}

Skin Toxicity in Patients Receiving Cet/RT

Effective management should address the epidermal barrier disruption, the inflammatory component and the possible superinfection risk as suggested by Bernier et al. [13].

Thus, in order to prevent epidermal barrier disruption, the following suggestion should be followed: (1) avoid potentially skin-drying habits/products (e.g. hot water, alcohol-based cosmetics). (2) Avoid synthetic soaps that might disrupt the liposoluble component of the epidermis barrier, eventually worsening xerosis. The affected area should not be washed more than twice a day using $\mathrm{pH} 5$ soap and/or showering oils for sensitive skin, followed by moisturizing with an unscented moisturizer recommended for dry skin [27]. (3) Use moisturizing products containing urea $(<3 \%)$ and/or a high glycerol content to prevent excessive skin water loss and consequently improve skin hydration $[25,27]$.

In order to delay skin exfoliation, according to Bernier et al. [13] and Pinto et al. [25], it is advisable to: (1) avoid skin irritants such as alcohol-based lotions and perfumes. (2) Avoid synthetic clothes; it is advisable to use only linen or cotton clothes on the irradiated areas. (3) Shave with a sharp disinfected wet razor to reduce the risk of folliculitis. (4) Use pre-shaving emollient creams and moisturizing after-shave.

When xerotic, inflamed skin begins to be covered firstly by thin scales and thereafter by yellow thick crusts, pain may be a consequence $[14,22]$ of skin microlesions due to hurting scales themselves. In this case, it is necessary to superinfection and bleeding and to help reduce pain [13, the damaged skin. Hydrogel can help to keep crusts flexible and to reduce the risk of skin trauma and pain. After having removed the crusts and cleaned the skin with Ringer's lactate or saline water, the injured skin needs to be loid does not interfere with high-energy X-radiation, preserving its build-up effect. In order not to traumatize the region with frequent medication, this dressing can be left on the site as long as possible ( 3 days to 1 week). If local (green/yellowish exudation) [15] or systemic [altered systemic inflammatory response syndrome (SIRS) parame- ters] infection [13] is suspected, topical and systemic antibiotics should be used after appropriate cultural swabs. Obviously, while local and systemic antibiotics effective against the causative pathogen are preferred for suspected cutaneous infection without altered SIRS parameters, in the case of SIRS, empirical systemic antibiotics need to be precociously started, as well as a successively modified antibiotic regimen based on microbiologic data [33]. Indeed, an infection associated with SIRS-altered parameters must be considered as sepsis [34] which needs to be treated with antibiotics as soon as possible [33].

In conclusion, it is recognized that the management of radiation dermatitis associated with Cet/RT is an evolving area, and it is likely that as our knowledge of the pathophysiological mechanism involved in these reactions improves, alternative management approaches may be considered.

The management of skin toxicity is important as it has a substantial impact on the quality of life and, potentially, on treatment outcome; hence, specific guideline formulation is strongly demanded to provide a systematic perspective on this issue and to avoid potential misinterpretations.

\section{Acknowledgement}

We thank Laurence Preston for his revision of the English language of the manuscript.

\section{Disclosure Statement}

The authors disclose no conflicts of interest. perform the debridement of crusts to reduce the risk of $15,21]$.

This procedure should be done without traumatizing protected by hydrocolloid films. An 'ultrathin' hydrocol-

References 
5 Berger B, Belka C: Severe skin reaction secondary to concomitant radiotherapy plus cetuximab. Radiat Oncol 2008;3:5.

-6 Merlano M, Russi E, Benasso M, Corvò R, Colantonio I, Vigna-Taglianti R, et al: Cisplatin-based chemoradiation plus cetuximab in locally advanced head and neck cancer: a phase II clinical study. Ann Oncol 2011;22: 712-717.

7 Rampino M, Bacigalupo A, Russi E, Schena $M$, Lastrucci L, Iotti C, et al: Efficacy and feasibility of induction chemotherapy and radiotherapy plus cetuximab in head and neck cancer. Anticancer Res 2012;32:195199.

8 Bölke E, Gerber PA, Lammering G, Peiper M, Müller-Homey A, Pape H, et al: Development and management of severe cutaneous side effects in head-and-neck cancer patients during concurrent radiotherapy and cetuximab. Strahlenther Onkol 2008;184: 105-110.

-9 Janko M, Ontiveros F, Fitzgerald TJ, Deng A, DeCicco M, Rock KL: IL-1 generated subsequent to radiation-induced tissue injury contributes to the pathogenesis of radiodermatitis. Radiat Res 2012;178:166-172.

10 Liu T, Gao Y-J, Ji R-R: Emerging role of Tolllike receptors in the control of pain and itch. Neurosci Bull 2012;28:131-144.

-11 Madison KC: Barrier function of the skin: 'la raison d'être' of the epidermis. J Invest Dermatol 2003;121:231-241.

-12 Albanell J, Rojo F, Averbuch S, Feyereislova A, Mascaro JM, Herbst R, et al: Pharmacodynamic studies of the epidermal growth factor receptor inhibitor ZD1839 in skin from cancer patients: histopathologic and molecular consequences of receptor inhibition. J Clin Oncol 2002;20:110-124.

$>13$ Bernier J, Russi EG, Homey B, Merlano MC, Mesía R, Peyrade F, et al: Management of radiation dermatitis in patients receiving cetuximab and radiotherapy for locally advanced squamous cell carcinoma of the head and neck: proposals for a revised grading system and consensus management guidelines. Ann Oncol 2011;22:2191-2200.

14 Peter RU, Beetz A, Ried C, Michel G, van Beuningen D, Ruzicka T: Increased expression of the epidermal growth factor receptor in human epidermal keratinocytes after exposure to ionizing radiation. Radiat Res 1993; 136:65-70.
15 Russi EG, Merlano MC, Comino A, Numico G: Ultrathin hydrocolloid dressing in skin damaged from alternating radiotherapy and chemotherapy plus cetuximab in advanced head and neck cancer (G.O.N.O. AlteRCC Italian Trial): in regard to Macmillan et al. (Int J Radiat Oncol Biol Phys 2007;68:864-872). Int J Radiat Oncol Biol Phys 2007;69:638-639.

16 Pastore S, Mascia F, Mariani V, Girolomoni G: The epidermal growth factor receptor system in skin repair and inflammation. J Invest Dermatol 2008;128:1365-1374.

17 Mascia F, Mariani V, Girolomoni G, Pastore $S$ : Blockade of the EGF receptor induces a deranged chemokine expression in keratinocytes leading to enhanced skin inflammation. Am J Pathol 2003;163:303-312.

18 Pastore S, Mascia F, Mariotti F, Dattilo C, Mariani V, Girolomoni G: ERK1/2 regulates epidermal chemokine expression and skin inflammation. I Immunol 2005; 174:5047-5056.

19 Sorensen OE: Injury-induced innate immune response in human skin mediated by transactivation of the epidermal growth factor receptor. J Clin Invest 2006;116:1878-1885.

20 Eilers RE, Gandhi M, Patel JD, Mulcahy MF, Agulnik M, Hensing T, et al: Dermatologic infections in cancer patients treated with epidermal growth factor receptor inhibitor therapy. J Natl Cancer Inst 2010;102:47-53.

21 Russi E, Merlano M, Numico G, Corvò R, Benasso $M$, Vigna-Taglianti $R$, et al: The effects on pain and activity of daily living caused by crusted exudation in patients with head and neck cancer treated with cetuximab and radiotherapy. Support Care Cancer 2012;20: 2141-2147.

22 Hymes SR, Strom EA, Fife C: Radiation dermatitis: clinical presentation, pathophysiology, and treatment 2006. J Am Acad Dermatol 2006;54:28-46.

23 Giro C, Berger B, Bölke E, Ciernik IF, Duprez $F$, Locati L, et al: High rate of severe radiation dermatitis during radiation therapy with concurrent cetuximab in head and neck cancer: results of a survey in EORTC institutes. Radiother Oncol 2009;90:166-171.

$>24$ Agero ALC, Dusza SW, Benvenuto-Andrade C, Busam KJ, Myskowski P, Halpern AC: Dermatologic side effects associated with the epidermal growth factor receptor inhibitors. J Am Acad Dermatol 2006;55:657-670.
25 Pinto C, Barone CA, Girolomoni G, Russi EG, Merlano MC, Ferrari D, et al: Management of skin toxicity associated with cetuximab treatment in combination with chemotherapy or radiotherapy. Oncologist 2011;16:228-238.

26 Lacouture ME, Maitland ML, Segaert S, Setser A, Baran R, Fox LP, et al: A proposed EGFR inhibitor dermatologic adverse event-specific grading scale from the MASCC skin toxicity study group. Support Care Cancer 2010;18: 509-522.

-27 Segaert S, Van Cutsem E: Clinical signs, pathophysiology and management of skin toxicity during therapy with epidermal growth factor receptor inhibitors. Ann Oncol 2005;16:1425-1433.

28 Common Terminology Criteria for Adverse Events (CTCAE) and Common Toxicity Criteria (CTC) v. 4. US Department of Health and Human Services, National Institutes of Health, National Cancer Institute, May 28, 2009 (cited July 10, 2010). http://evs.nci.nih. gov/ftp1/CTCAE/About.html

-29 Chen AP, Setser A, Anadkat MJ, Cotliar J, Olsen EA, Garden BC, et al: Grading dermatologic adverse events of cancer treatments: the Common Terminology Criteria for Adverse Events Version 4.0. J Am Acad Dermatol 2012;67:1025-1039.

30 Russi EG, Numico G, Merlano MC, Pinto C: Cetuximab-related radiation dermatitis in head and neck cancer patients: in regard to Studer et al. (Int J Radiat Oncol Biol Phys in press). Int J Radiat Oncol Biol Phys 2011;79:1278.

31 Studer G, Brown M, Salgueiro EB, Schmückle $\mathrm{H}$, Romancuk N, Winkler G, et al: Grade 3/4 dermatitis in head and neck cancer patients treated with concurrent cetuximab and IMRT. Int J Radiat Oncol 2011;81:110-117.

-32 Russi EG, Bensadoun RJ, Merlano MC, Bourhis J, Ricardi U, Giralt J, et al: Bio-radiation dermatitis: the need of a new grading: in regard to Bernier et al. (Ann Oncol 2011;22: 2191-2200). Ann Oncol 2013;24:2463-2465.

33 Barie PS, Eachempati SR: What is the role of empirical antibiotic therapy in sepsis? in Deutschman CS, Neligan PJ (eds): EvidenceBased Practice of Critical Care. Philadelphia, Saunders/Elsevier, 2010, pp 182-197.

-34 Levy MM, Fink MP, Marshall JC, Abraham E Angus D, Cook D, et al: 2001 SCCM/ESICM/ ACCP/ATS/SIS International Sepsis Definitions Conference. Intensive Care Med 2003; 29:530-538.
Biologically Optimized Radiation

Treatment Toxicity Management
Oncology 2013;85:257-261 DOI: $10.1159 / 000355579$ 